\title{
Magnetic and Mechanical Analysis of the HQ Model Quadrupole Designs for LARP
}

\author{
Helene Felice, Shlomo Caspi, Paolo Ferracin, Vadim Kashikhin, Igor Novitski, GianLuca Sabbi and \\ Alexander Zlobin
}

\begin{abstract}
Insertion quadrupoles with large bore and high gradient are required to upgrade the luminosity of the Large Hadron Collider (LHC). The US LHC Accelerator Research Program is developing $\mathrm{Nb}_{3} \mathrm{Sn}$ technology for the upgrade. This effort includes a series of $1 \mathrm{~m}$ long Technology Quadrupoles (TQ), to demonstrate the reproducibility at moderate field, and High-gradient Quadrupoles (HQ) to explore the magnet performance limits in terms of peak fields, forces and stresses. The HQ models are expected to achieve peak fields of $15 \mathrm{~T}$ or higher. A coil aperture of $90 \mathrm{~mm}$, corresponding to gradients above $300 \mathrm{~T} / \mathrm{m}$, was chosen as the baseline. Peak stresses above $150 \mathrm{MPa}$ are expected. Progress on the magnetic and mechanical design of the HQ models will be reported.
\end{abstract}

Index Terms-Superconducting accelerator magnets, $\mathrm{Nb}_{3} \mathrm{Sn}$, IR quadrupoles

\section{INTRODUCTION}

$\mathrm{R}$ EPLACING the first-generation LHC interaction region quadrupoles with higher performance magnets is one of the steps toward a $10^{35} \mathrm{~cm}^{-2} \mathrm{~s}^{-1}$ luminosity. Among the solutions considered, a possible upgrade relies on the $\mathrm{Nb}_{3} \mathrm{Sn}$ technology.

The LHC Accelerator Research Program (LARP), started in 2004 and based on a coordinated US effort, is developing $\mathrm{Nb}_{3} \mathrm{Sn}$ prototype quadrupoles for the interaction region. In order to demonstrate the feasibility and effectiveness of the $\mathrm{Nb}_{3} \mathrm{Sn}$ technology, several series of magnets are being designed, built and tested. The first series of magnets consists of the "Technology Quadrupoles" (TQ): 1-meter long $90 \mathrm{~mm}$ aperture quadrupoles, producing a gradient in the range of 200-230 T/m [1]-[2]. The following step in the LARP program, starting in 2008, is the scale-up of the TQ series to a 4-meter "Long Quadrupole" (LQ) series [3]. The objectives of the High gradient Quadrupole (HQ) are to explore the field, forces and stress limits of the $\mathrm{Nb}_{3} \mathrm{Sn}$ technology. A peak field above $15 \mathrm{~T}$ is targeted as well as a gradient up to $300 \mathrm{~T} / \mathrm{m}$ in a $90 \mathrm{~mm}$ bore. These high magnetic performances are

Manuscript received August 28, 2007. This work was supported by the Director, Office of Energy Research, Office of High Energy and Nuclear Physics, High Energy Physics Division, U.S. Department of Energy, under contract No. DE-AC02-05CH11231.

H.Felice, S. Caspi, P, Ferracin and GL Sabbi are with Lawrence Berkeley National Laboratory, Berkeley, CA 94720 USA (e-mail: HFelice@lbl.gov).

V. Kashiskhin, I. Novitski and A.Zlobin are with Fermilab National Accelerator Laboratory, Batatvia, IL 60510-0500 USA. generating stresses above $180 \mathrm{MPa}$ in the conductor, making the mechanical structure design particularly challenging. This paper presents a summary of the magnetic configurations considered and detailed mechanical study.

\section{MAGNeTiC DeSign}

The HQ models will investigate the limits of $\mathrm{Nb}_{3} \mathrm{Sn}$ in term of peak field, forces and stresses. To meet these requirements, several layouts and apertures can be considered.

One approach consists in generating a gradient up to 300 $\mathrm{T} / \mathrm{m}$ in a $90 \mathrm{~mm}$ aperture. Within LARP, developing a $90 \mathrm{~mm}$ aperture HQ is a cost-effective way to explore high gradient and high stress quadrupoles with the possibility to share some tooling and coils with the TQ series. A magnetic design study has been performed previously and reported in [4]. Two crosssections were selected: HQ1 and HQ2, presented in Fig. 1 (a) and (b).

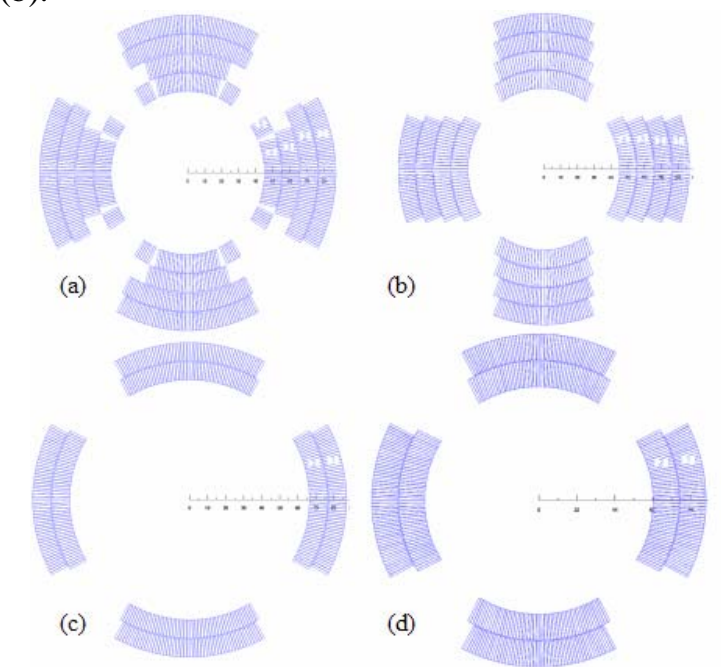

Fig. 1. HQ cross-section. (a) HQ1 - (b) HQ2 - (c) HQ1out - (d) HQ1out

Both of them have a four-layer layout. In HQ1, the inner double-layer is identical to the TQ model magnet. The outer double-layer has been optimized to minimize the $b_{6}$ multipole component. Since both double-layers feature a similar peak field, grading is not possible and the TQ strand and cable are used everywhere with a slightly different keystone angle in each double-layer. In HQ2, which was optimized to maximize the gradient without the constraint of using the TQ coils, the outermost layers are experiencing a lower field. The grading of the conductor is then possible with the use of a larger diameter strand in the inner double-layer. Tables I and II report the conductor and coil parameters as well as the 
expected performance at the operation temperature $T_{\mathrm{op}}=1.9 \mathrm{~K}$ and for a gradient of $300 \mathrm{~T} / \mathrm{m}$, chosen to compare the two designs. The critical current density taken into account in the computation is $3000 \mathrm{~A} / \mathrm{mm}^{2}$ at $12 \mathrm{~T}$ and $4.2 \mathrm{~K}$. The yoke is located at $10 \mathrm{~mm}$ from the coils, based on preliminary studies on saturation effects [4], and its outer radius is equal to 250 $\mathrm{mm}$. A larger outer radius does not impact the magnetic behavior of the magnet but could be implemented for mechanical reasons. The reference radius $\mathrm{R}_{\mathrm{ref}}$ is taken at half of the aperture.

A second approach in exploring the limit of $\mathrm{Nb}_{3} \mathrm{Sn}$ magnets is to consider large aperture quadrupoles. Indeed, recent analysis [5] indicates that the optimal aperture for the full upgrade of the LHC is in the range of 100 to $130 \mathrm{~mm}$. A first step toward $130 \mathrm{~mm}$ aperture quadrupoles could be the test of the outer double layer of the HQ1 in standalone configuration. Because of the different optimization process, the HQ1 outer double-layer shows considerably better field quality.

TABLE I CONDUCTOR, CABLE AND COIL PARAMETERS

\begin{tabular}{lccccc}
\hline \multirow{2}{*}{\multicolumn{1}{c}{ Parameter }} & \multirow{2}{*}{ Units } & \multirow{2}{*}{ HQ1 } & \multicolumn{2}{c}{ HQ2 } & \multirow{2}{*}{ HQ1out } \\
& & in/out & inner & outer & \\
\hline Strand diameter & $\mathrm{mm}$ & 0.7 & 0.85 & 0.7 & 0.7 \\
Cu/non-Cu ration & & 0.87 & 0.87 & 0.87 & 0.87 \\
Number strands & & 27 & 23 & 27 & 41 \\
Cable width (bare) & $\mathrm{mm}$ & 10.05 & 10.05 & 10.05 & 15.1 \\
Mid-thickness (bare) & $\mathrm{mm}$ & 1.26 & 1.54 & 1.26 & 1.275 \\
Keystone angle & $\mathrm{deg}$ & $1 / 1.13$ & 1.40 & 1.13 & 1 \\
Insulation thickness & $\mathrm{mm}$ & 0.125 & 0.125 & 0.125 & 0.125 \\
Number of turns & & $34 / 52$ & 32 & 38 & 54 \\
\hline \hline
\end{tabular}

TABLE II MAGNET PARAMETERS

\begin{tabular}{|c|c|c|c|c|c|}
\hline Parameter & Units & HQ1 & HQ2 & HQ1out & HQ1out ${ }^{*}$ \\
\hline Short sample gradient & $\mathrm{T} / \mathrm{m}$ & 312 & 319 & 185 & 204 \\
\hline $\begin{array}{l}\text { Short sample current } \\
\mathrm{I}_{\mathrm{ss}}\end{array}$ & $\mathrm{kA}$ & 10.6 & 12.45 & 13.5 & 16.5 \\
\hline Coil peak field at $\mathrm{I}_{\mathrm{ss}}$ & $\mathrm{T}$ & 15.74 & 16.03 & 14.53 & 15.59 \\
\hline $\begin{array}{l}\text { Comparison gradient } \\
G_{\text {comp }} \text { at } T_{\text {op }} \\
\text { Results at } \mathbf{G}_{\text {comp }}\end{array}$ & $\mathrm{T} / \mathrm{m}$ & 300 & 300 & 185 & 185 \\
\hline Current & kA & 10.2 & 11.65 & 13.5 & 14.8 \\
\hline Inductance & $\mathrm{mH} / \mathrm{m}$ & 26.1 & 19 & 12.1 & 12.1 \\
\hline Energy & $\mathrm{MJ} / \mathrm{m}$ & 1.36 & 1.3 & 1.1 & 1.3 \\
\hline X Lorentz force/octant & $\mathrm{MN} / \mathrm{m}$ & 3.9 & 3.4 & 2.7 & 3.1 \\
\hline YLorentz force/octant & $\mathrm{MN} / \mathrm{m}$ & -4.9 & -4.6 & 3.5 & -3.9 \\
\hline Dodecapole at $\mathrm{R}_{\text {ref }}$ & $\mathrm{b}_{6}$ & $0.28^{\mathrm{a}}$ & $0.6^{\mathrm{a}}$ & $3.97^{\mathrm{b}}$ & $0.28^{\mathrm{b}}$ \\
\hline 10 -pole at $R_{\text {ref }}$ & $\mathrm{b}_{10}$ & $-0.05^{\mathrm{a}}$ & $-0.9^{\mathrm{a}}$ & $-2.37^{\mathrm{b}}$ & $-1.76^{\mathrm{b}}$ \\
\hline
\end{tabular}

A third option, HQ1out ${ }^{*}$, has been investigated for the 130 mm quadrupoles and is presented in Fig.1 (d). The purpose of this design is to increase the gradient and verify possible advantages given by a wider cable in term of stress and quench protection. The coil layout is comparable to HQ1out with a pole angle of $30^{\circ}$. The conductor parameters are described in Table I. The expected performances of HQ1out and HQ1out ${ }^{*}$ are summarized in Table II. The comparison is made for $185 \mathrm{~T} / \mathrm{m}$, the short-sample gradient of HQ1out.

From this study, it appears that several solutions are possible for the magnetic layout of HQ. Regarding the four layer configurations, HQ2 produces the highest gradient but requires the use of different strands for the inner and outer layer. HQ1 relies only on the TQ cable and has the advantage to be a "2-in-1" configuration since the outer double layer can be tested in stand-alone configuration. Finally, HQ1out ${ }^{*}$ focuses the study on large aperture quadrupoles, producing a higher gradient than HQ1out at short sample. Stress comparison will be covered in the next section.

\section{MECHANICAL ANALYSIS}

\section{A. Mechanical design}

Within LARP, two different mechanical design concepts are under study with the TQ series. The first one relies on stainless steel collars, iron yoke and welded stainless steel skin and is presently implemented in the TQC magnets ([1]-[6]). In this concept, all the pre-stress is applied to the coil during the collaring and the yoking process, at room temperature. The extension of this concept to the long-scale magnet has already been demonstrated with $\mathrm{NbTi}$ magnets, as well as the alignment of the coils. The second concept, used in the TQS magnets ([2]-[7]), is based on an aluminum shell surrounding an iron yoke and support pads. In this design, a small amount of the preload (between 10 and $40 \%$ ) is applied to the coil at room temperature by the bladder and key operation [8]. The main part of the pre-stress is then provided during cool-down by the shrinkage of the outer aluminum shell. The extension to long length is being investigated with the LR magnet series [9]. Alignment features have still to be implemented.

In the HQ design, the Lorentz forces require a coil preload of $130 \mathrm{MPa}$. For a 4-layer configuration, this represents a force of the order of $5 \mathrm{MN} / \mathrm{m}$ per octant, which is twice the value involved in TQ. This requirement leads to the choice of a shell-based structure since the amount of force applied to the coil can be controlled by the shell thickness and is achievable for the HQ configuration. The structure has to provide support to the magnet at $1.9 \mathrm{~K}$ for a gradient of $300 \mathrm{~T} / \mathrm{m}$ or $185 \mathrm{~T} / \mathrm{m}$. Alignment features are also implemented.

\section{B. TQS-based structure}

\section{1) Components}

The first mechanical structure considered is based on the TQS02 design [7] and is shown in Fig.3. The coils are wound around titanium islands. A slot in the pole has been added to decrease the peak stress in the inner layer pole turns of the coil after cool-down. The aluminum shell is $40 \mathrm{~mm}$ thick, the outer radius of the yoke is equal to $310 \mathrm{~mm}$ and the pad thickness is $40 \mathrm{~mm}$. Four holes drilled in the pads provide room for aluminum rods applying the axial preload. Two $15 \mathrm{~mm}$ wide 5 $\mathrm{mm}$ thick nominal keys are positioned at $15 \mathrm{~mm}$ from the midplane. The pads are made of iron because of the magnetic design and an alignment feature replaces the TQS iron fillers located between the coils and the pads. Its purpose is to align the coils with the pads.

From a mechanical analysis standpoint, this component behaves like a solid ring. In order to minimize the saturation effect by maintaining the appropriate distance between the coils ad the iron parts, the ring has a thickness of $10 \mathrm{~mm}$. 
From the assembly viewpoint, several solutions can be considered. This alignment feature can be a thin collar or can be split into four $90^{\circ}$ parts. Fig. 4 shows two different solutions considered for the 4 parts case, both including alignment keys between the coil and the ring and between the ring and the pad: a "mid-plane to mid-plane" configuration (left), where each part is placed in between two coil midplanes, and a "pole-to-pole" configuration (right), with the same parts rotated of $90^{\circ}$.

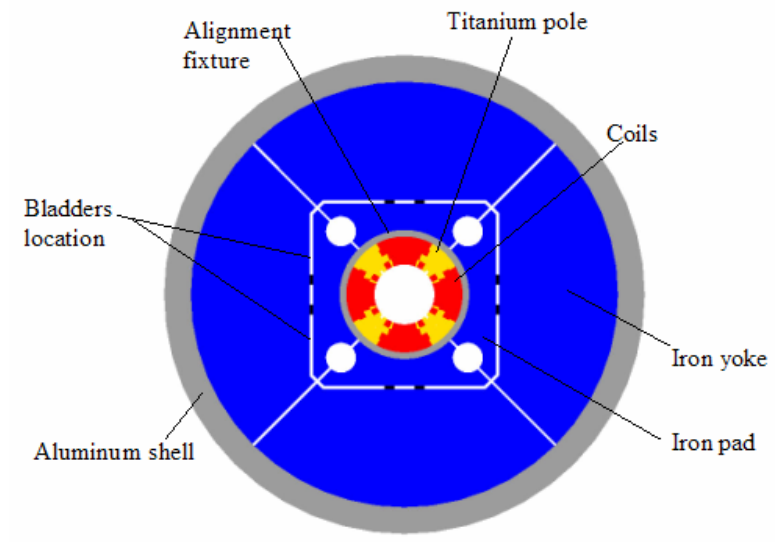

Fig. 3. Cross-section of HQ1 with the TQS-based structure
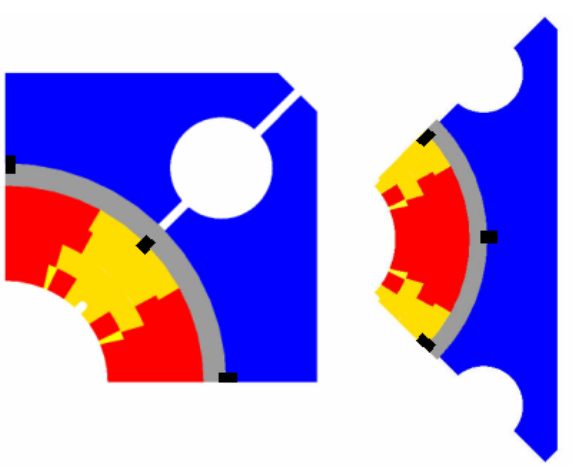

Fig. 4. Alignment feature: left) mid-plane to mid-plane configuration - right) pole to pole configuration - the black rectangles represent the alignment key between the ring and the pole and between the ring and the pad

The preload is applied at room temperature by eight $60 \mathrm{~mm}$ bladders located outside the key span as indicated in Fig. 3. Their pressurizati opens a gap between the nominal keys and the yoke allowing the insertion of some interference shims (up to $0.5 \mathrm{~mm}$ ) defining the coil pre-stress before cool-down. The loading feature consisting of the nominal keys and the interference shims is called interference keys.

\section{2) Analysis}

One octant of each magnet has been analyzed in 2D with ANSYS, with a friction coefficient of 0.2. The first step of the computation simulates the state of the structure after insertion of the interference shims, by introducing a positive interference between the nominal keys and the yoke. The second step is the cool-down and the final step takes into account the Lorentz forces computed with an ANSYS 2D FEM model for a gradient of $300 \mathrm{~T} / \mathrm{m}$ or $185 \mathrm{~T} / \mathrm{m}$.

All the contact elements between the components allow sliding and separation except the contact element between the two double-layer and the pole/coil contacts, which are glued. Consequently, when the Lorentz forces are applied, a lack of preload results in a tensile stress at the pole-coil contact. In order to reduce the azimuthal stress in the winding, we assumed that the epoxy can withstand a maximum tension of $20 \mathrm{MPa}$ at $300 \mathrm{~T} / \mathrm{m}$ or $185 \mathrm{~T} / \mathrm{m}$. Regarding the boundary conditions, zero displacement is imposed on the mid-plane and at $45^{\circ}$ to the pole and to the shell. The same conditions are imposed to the alignment feature, whatever configuration is considered (collars or $90^{\circ}$ parts). Compression has to be ensured at the mid-plane (respectively at the pole) in the "midplane to mid-plane" (respectively in the "pole-to-pole") configuration.

Several materials have been considered for the alignment feature. With stainless steel, due to its stiffness, a $10 \mathrm{~mm}$ feature takes $35 \%$ of the preload applied during bladder operation and cool-down. On the contrary, an aluminum feature takes only $5 \%$ of the preload. That is why, in the results presented here, the aluminum has been chosen. With this material, the alignment feature is in tension at the pole (25 $\mathrm{MPa}$ ) and in compression on the mid-plane (-45 MPa), imposing the "mid-plane to mid-plane" configuration or a collar-type feature.

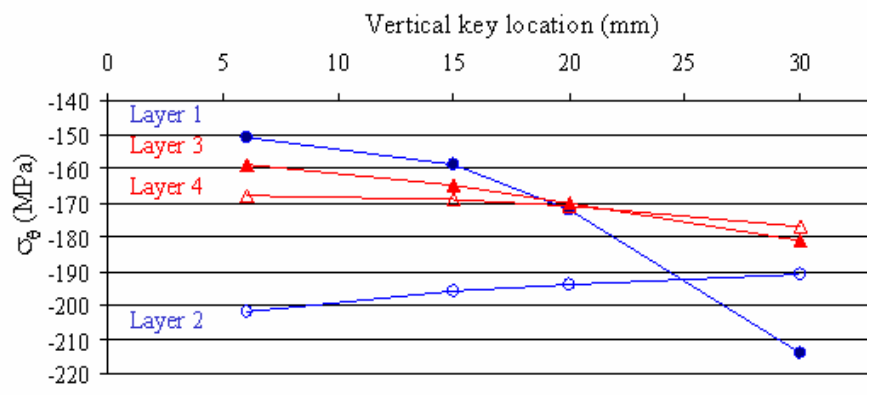

Fig. 5. Variation of the maximum azimuthal stress in MPa for a gradient of $300 \mathrm{~T} / \mathrm{m}$ of the four layers of HQ1 versus the vertical interference key location in $\mathrm{mm}$. There is pole /coil contact for each vertical key location. The dot curve corresponds to the inner double layer and the triangle to the outer double layer. The blank symbols are for the outermost layer of each double layer and the full symbols are for the innermost layer of each double layer

The vertical interference key location, which corresponds to the distance of the key with respect to the mid-plane, has a major role in the coil stress distribution. Fig. 5 shows the variation of the maximum azimuthal stress in the different layers of HQ1 versus the vertical key location. For each vertical key location, the computations have been carried out for a gradient of $300 \mathrm{~T} / \mathrm{m}$ and the interference has been chosen allowing $20 \mathrm{MPa}$ of tension in the pole. For the innermost layer, the compressive stress increases strongly when the vertical position of the key increases because the coil is less supported radially. Consequently, the radial component of the Lorentz forces induces the bending of the coil in the midplane. At the same time, layers 3 and 4 see their stress increasing due to the accumulation of the preload in the outer layers. A location at $15 \mathrm{~mm}$ from the mid-plane has been chosen. In addition, it appeared that if a wide key, centered on the mid-plane and going up to the optimized location, is used instead of two, the same stress distribution is computed in the coil. Therefore, a $60 \mathrm{~mm}$ key centered on the mid-plane could be used.

The second important parameter is the thickness of the pad. Its bending is used to provide the pre-stress to all the layers of the magnet, in particularly to the innermost layer. If the pad is 
too thick, it cannot bend and the preload is accumulated in the outer layers. An increase of the interference will only result in an increase of the compressive stress in the outer layers. On the contrary, if the pad is too thin, its bending stress will exceed the maximum tensile stress at cryogenic temperature for brittle material like iron. These considerations lead to a pad thickness of $40 \mathrm{~mm}$.

The stress distribution for each step of the computation is summarized in Table III for HQ1, HQ2, HQ1out and HQ1out*. The highest value of the maximum azimuthal stress $\sigma_{\theta \max }$, (about $196 \mathrm{MPa}$ ) is reached in the HQ1 design during excitation. Although this value is well above the degradation limit of $150 \mathrm{MPa}$ currently assumed for the $\mathrm{Nb}_{3} \mathrm{Sn}$ conductor, it might be tolerated because it occurs in a lowfield region (below $9 \mathrm{~T}$ ). The tension in the iron pad after cool-down, corresponding to the first principal stress $\sigma_{1} \max$, remains safely below $200 \mathrm{MPa}$ in all cases. For the $130 \mathrm{~mm}$ aperture magnets, a peak stress of -193 MPa occurs in HQ1out at $185 \mathrm{~T} / \mathrm{m}$, whereas, lower stresses are estimated for HQ1out ${ }^{*}$ at the same gradient. For both of them, the entire preload is given by the shrinkage of the $40 \mathrm{~mm}$ aluminum shell. In order to allow the use of bladders and extend the range of the possible preload, a thinner shell can be considered for these magnets.

TABLE III MECHANICAL ANALYSIS RESULTS

\begin{tabular}{lccccc}
\hline \hline \multicolumn{1}{c}{ Parameter } & Units & HQ1 & HQ2 & HQ1out & $\begin{array}{c}\text { HQ1out } \\
-15\end{array}$ \\
\hline Gradient & T/m & 300 & 300 & 185 & 185 \\
Interference & micron & 450 & 420 & 0 & 50 \\
COIL & & & & & \\
$\sigma_{\theta \text { max }}$ interference & $\mathrm{MPa}$ & -61 & -55 & 0 & -8 \\
$\sigma_{\theta \max }$ cool-down & $\mathrm{MPa}$ & -175 & -169 & -151 & -133 \\
$\sigma_{\theta \max }$ Lorentz forces & $\mathrm{MPa}$ & -196 & -172 & -193 & -147 \\
SHELL & & & & & \\
$\sigma_{\theta}$ interference & $\mathrm{MPa}$ & 62 & 60 & 0 & 6 \\
$\sigma_{\theta \max }$ cool-down & $\mathrm{MPa}$ & 166 & 155 & 80 & 89 \\
PAD & & & & & \\
$\sigma_{1 \text { max }}$ cool-down & $\mathrm{MPa}$ & 175 & 164 & 120 & 140 \\
\hline \hline
\end{tabular}

\section{Decoupling structure}

The use of two bladders is a major difference with the previous LBNL magnets, which relied on a unique bladder per quadrant centered on the mid-plane between the two nominal keys [2]. Using two bladders and moving them outside the key span modify the stress distribution in the coil during bladder operation, preventing the coil from being supported radially. In HQ1, the bending of the inner layer leads to a compressive stress on the mid-plane of $-120 \mathrm{MPa}$, which could be avoided by partially decoupling the coil from the pad. A solution is proposed in Fig. 6. The outer diameter $(700 \mathrm{~mm})$, the poles material and the alignment feature remain identical, but the pad and the yoke have been modified. In order to decouple the coil from the bending of the pad during bladder operation, the bladders have to be taken away from the coil. At the same time, the optimum pad thickness and the optimized key dimension established previously have to be maintained. To reach this goal, a bigger pad has been considered. In order to understand its mechanical behavior, it can be broken up in two different parts (Fig. 6). The first one is the active part (40 mm thick), which is close to the coil. The second part is a passive part and is providing room for the bladders. It can bend without damaging the coils because of the small cut separating it from the active part. The location of the new nominal key has been optimized and is equal to $20 \mathrm{~mm}$. The preload is transmitted from the passive pad to the active pad via a $30 \mathrm{~mm}$ bridge, whose the dimension corresponds to the optimized location of the key in the TQS-based structure described in section $\mathrm{B}$.

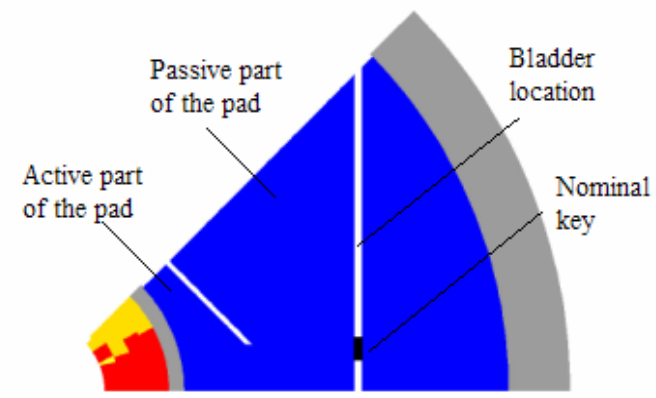

Fig. 6. One octant of the decoupling structure for HQ1

The stress distribution in the coil for this configuration is identical to the stress distribution computed with the TQSbased structure. The difference occurs during bladder operation (eight $100 \mathrm{~mm}$ bladders in total pressurized, at 35 $\mathrm{MPa}$ ) where the compressive stress on the mid-plane is lowered to $-12 \mathrm{MPa}$ and the peak stress is equal to $-100 \mathrm{MPa}$ and occurs in a low field region.

\section{CONCLUSION}

Progress in the design study of the HQ model quadrupole for LARP was presented. Several magnetic designs were described and some mechanical analysis results detailed. Further studies will have to introduce quench analysis, to optimize the mechanical structure and to define the assembly procedure.

\section{REFERENCES}

[1] S. Feher et al, "Development and Test of LARP Technological Quadrupole (TQC) Magnet”, IEEE Trans. Applied Supercond, vol 17, No 2, June 2007, pp. 1126-1129.

[2] S. Caspi et al, "Fabrication and Test TQS01 - A 90 mm Nb3Sn Quadrupole Magnet for LARP”, IEEE Trans. Applied Supercond, vol 17, No 2, June 2007, pp. 1122-1125.

[3] G. Ambrosio et al, "LARP Long Nb3Sn Quadrupole Design Study", this conference.

[4] GL. Sabbi et al., "Design Studies of Nb3Sn High-Gradient Quadrupole Models for LARP”, IEEE Trans. Applied Supercond, vol 17, No 2, June 2007, pp. 1051-1054.

[5] J.P. Koutchouk, "Investigations of the Parameter Space for the LHC Luminosity Upgrade”, EPAC’06, Edinburgh, UK, June 26-30, 2006

[6] R. Bossert et al., "Development and Test of TQC02, LARP Technological Quadrupole Magnet”, this conference

[7] S. Caspi et al., "Test results of TQS02 - a Second Nb3Sn Quadrupole Magnet Model for LARP”, this conference

[8] S. Caspi et al, "The Use of Pressurized Bladders for Stress Control of Superconducting Magnets", IEEE Trans on Applied Supercond, vol 11, No 1, March 2001, pp. 2272 - 2275

[9] P. Ferracin et al., "Assembly and Test of a Support Structure for $3.6 \mathrm{~m}$ Long $\mathrm{Nb}_{3} \mathrm{Sn}$ Racetrack Coils", this conference. 